\title{
Alexis Fritz, Christof Mandry, Ingo Proft, Josef Schuster (Hrsg) (2021) Digitalisierung im Gesundheitswesen. Anthropologische und ethische Herausforderungen der Mensch-Maschine-Interaktion
}

\author{
Jahrbuch für Moraltheologie, Bd. 5, Herder, Freiburg i.Br. u.a., \\ 248 Seiten, $40 €$, ISBN 978-3451387647
}

\section{Hermann Diebel-Fischer}

Angenommen: 15. Dezember 2021 / Online publiziert: 3. Januar 2022

(C) Der/die Autor(en) 2021

Gesundheit zu erhalten oder wiederherzustellen, ist die Kernaufgabe des Gesundheitswesens, die unter monetären Restriktionen und einer zunehmenden Personalknappheit erfüllt wird. Das Transformationsprojekt der Digitalisierung, das momentan stattfindet, erfasst in diesem Zusammenhang alle Teilbereiche des Gesundheitswesens und geht mit Effektivitäts- und Effizienzsteigerungsversprechen einher, die indem sie auch den Restriktionen begegnen - einen Raum für ethische wie anthropologische Rückfragen eröffnen. Von diesen wird im vorliegenden Band das Gebiet der Mensch-Maschine-Interaktion (MMI) herausgegriffen und beleuchtet - sowohl im Hinblick auf Grundlagenfragen (Teil I) als auch mit Blick auf ausgewählte Praxisfelder (Teil II).

Unter dem Titel Science or Fiction eröffnet Ingo Proft die Debatte und plädiert hinsichtlich der Anforderungen an eine KI im Gesundheitswesen für eine ,menschenfreundliche KI“, die sachorientiert, ergänzend und unterstützend arbeitet und in die der Mensch immer noch steuernd eingreifen kann (S. 35-37). Ähnliches fordert Josef Schuster für Pflegeroboter (S. 224-226), ergänzt um das Recht darauf, auf diese Technik verzichten zu dürfen (S. 230). Auch Andreas Lob-Hüdepohl thematisiert - anhand von Exklusions- und Inklusionsmechanismen von Sensorprothetik die Möglichkeit eines Verzichts auf Technik: als Voraussetzung für die Souveränität der Betroffenen (S. 177f.). Souveränität, so Joachim Sautermeister in seinem Beitrag, könnten die Behandelten, die mit den Behandelnden gemeinsam entscheiden sollen, unter den Bedingungen der Digitalisierung nur erlangen, wenn sie „Gesundheitskompetenz" erwerben würden (S. 121 u. 130-134). Für Proft stellt sich mit Blick auf die Zukunft die Frage, ob weiterhin ein ,kategorialer Unterschied“ zwischen

Dr. Hermann Diebel-Fischer $(\bowtie)$

Technische Universität Dresden - ScaDS.AI, Dresden, Deutschland

E-Mail: hermann.diebel-fischer@tu-dresden.de 
Mensch und Maschine „,bestehen soll(te)“ (S. 39) - was die anderen Autor:innen mit großer Wahrscheinlichkeit bejahen würden:

Christof Mandry beleuchtet das Selbst vor dem Hintergrund der tiefen Hirnstimulation und fragt, welche Konsequenzen aus der Steuerbarkeit von Bewusstsein, Gefühlen und Bewegungen erwachsen. Technik werde zwar als entfremdend und dem Menschen äußerlich erfahren, sei aber gleichwohl sein Produkt, weswegen sie ihm eigentlich nicht fremd sei (S. 179 u. 197f.). Mit Blick auf die Pflegerobotik thematisiert Josef Schuster auch die Nähe bzw. Ferne von Mensch und Maschine: Es gebe zwar einen möglichen Anpassungsbedarf des Menschen an die Technik, Schuster votiert aber ,[p]rinzipiell“ für die Anpassung der Technik an den Menschen (S. 228f.). Franz-Josef Bormann diskutiert die MMI anhand der Frage, ob die praktische Vernunft durch eine KI ersetzt werden könne. Zwar bestünden schlagkräftige Einwände dagegen (S. 53-61); gleichwohl weist er auf die Leistungsfähigkeit von KI-Systemen hin, die den Menschen sinnvoll ergänzen können - solange diese nicht ihr (ärztliches) Erfahrungswissen aufgrund der Anwendung dieser Systeme einbüßten (S. 61-63).

Dieses Wissen spielt auch im Beitrag von Alexis Fritz und Lorina Buhr eine Rolle: Sie fragen - geleitet von der These, dass moderne bildgebende Verfahren einen Einfluss auf das, was als Gesundheit bzw. Krankheit gesehen wird, auf die MMI und somit auch auf die „Praxis des Sehens“ haben (S. 65) - nach der Normativität, die durch diese Verfahren erzeugt wird. Sie kommen zum Fazit, dass die subjektive Wahrnehmung und nicht die technische Bildgebung zur Rechtfertigung gedrängt werde (S. 88). Eine problematische Normativität eignet nach LobHüdepohl auch dem Konzept der Normalität, die deswegen als anzustrebendes Ideal (miss)verstanden werde (S. 165f.). Ergänzend dazu beobachtet Katharina Klöcker die „Prämissen und Paradigmen der Prädiktion“. Es besteht ihr zufolge die Gefahr, dass unter den Bedingungen der Digitalisierung medizinisch nur jenes als bedeutend wahrgenommen werde, was sich mit Daten darstellen lasse (S. 153). Deswegen plädiert sie dafür, jene Perspektiven, die mit Technik gar nicht oder nicht ausreichend erfasst werden, in den Blick zu nehmen (S. 157).

Anna Puzio und Alexander Filipović widmen sich auf informationsethischer Grundlage dem Problem der datafizierten, zergliederten Person als Resultat einer Digitalisierung des Gesundheitswesens und formulieren ,theologische Leitperspektive[n]", die die menschliche Identität vor diesem Hintergrund wahren helfen sollen: Man könne (a.) mit Bezug auf Henning Luther von einer ,fragmentarischen Identität“" ausgehen, die von einem ehemals Ganzen der Identität ausgeht und weiterhin darauf verwiese; mit der (b.) gleichzeitig die Fragilität des Geschöpfes adressiert würde, ohne dabei eine vollkommene Identität als Ideal zu fordern. Ferner soll (c.) Gesundheit als „Makrophänomen“ verstanden werden, d.h. dass sie nicht nur durch eine datafizierende Zergliederung des Menschen, sondern gerade durch den Blick aufs Ganze erreicht werden könne (S. 111-113).

Für die Fortschreibung normativer Ethik im Gesundheitswesen unter der Bedingung eines beschleunigten Fortschritts ergibt sich als methodische wie inhaltliche Konsequenz, dass es einer ,,[p]rospektive[n] Ethik“ (Klöcker, S. 138f.) und ,provisorische[n] Ethik“ (Schuster, S. 228) bedarf. 
In kritischer, aber nicht alarmistischer Art - und durchgängig mit einer Unterstreichung der Bedeutung des Menschen - setzen sich die Beitragenden mit relevanten Fragen auseinander. Dass der Mensch im Mittelpunkt steht und stehen soll, bildet das normative Fundament dieses Bandes - gleichzeitig wird damit kein technikfeindlicher, sondern ein vorsichtig-technikoptimistischer Grundton angeschlagen und auf moralisierend-belehrende und vorschnelle Bewertungen verzichtet.

Die theologische Perspektive ist immer sachbezogen, sodass erfreulicherweise eine diesseitsorientierte Debatte erfolgt. Insofern stellen die Autor:innen auch noch einmal die Bedeutung eines geisteswissenschaftlichen Beitrags zur Debatte um die voranschreitende Technisierung im Gesundheitswesen heraus. Welche Folgen solch ein multidisziplinärer Zugang für die Praxis haben wird, bleibt indes abzuwarten.

Der rezensierte Sammelband dürfte von allen, die sich mit dem Thema befassen, mit Gewinn gelesen werden.

Funding Open Access funding enabled and organized by Projekt DEAL.

Open Access Dieser Artikel wird unter der Creative Commons Namensnennung 4.0 International Lizenz veröffentlicht, welche die Nutzung, Vervielfältigung, Bearbeitung, Verbreitung und Wiedergabe in jeglichem Medium und Format erlaubt, sofern Sie den/die ursprünglichen Autor(en) und die Quelle ordnungsgemäß nennen, einen Link zur Creative Commons Lizenz beifügen und angeben, ob Änderungen vorgenommen wurden.

Die in diesem Artikel enthaltenen Bilder und sonstiges Drittmaterial unterliegen ebenfalls der genannten Creative Commons Lizenz, sofern sich aus der Abbildungslegende nichts anderes ergibt. Sofern das betreffende Material nicht unter der genannten Creative Commons Lizenz steht und die betreffende Handlung nicht nach gesetzlichen Vorschriften erlaubt ist, ist für die oben aufgeführten Weiterverwendungen des Materials die Einwilligung des jeweiligen Rechteinhabers einzuholen.

Weitere Details zur Lizenz entnehmen Sie bitte der Lizenzinformation auf http://creativecommons.org/ licenses/by/4.0/deed.de.

Interessenkonflikt H. Diebel-Fischer gibt an, dass kein Interessenkonflikt besteht. 